\title{
Evaluation of an Automated Dispensing Cabinet in Paediatric Intensive Care - Focus on Controlled Medications
}

\author{
Valentina LICHTNER ${ }^{\mathrm{a}, \mathrm{b}, 1}$, Mirela PRGOMET ${ }^{\mathrm{b}}$, Peter GATES $^{\mathrm{b}}$, \\ Bryony Dean FRANKLIN ${ }^{\mathrm{a}, \mathrm{c}}$ and Johanna I WESTBROOK ${ }^{\mathrm{b}}$ \\ ${ }^{a}$ Department of Practice and Policy, UCL School of Pharmacy, United Kingdom \\ ${ }^{\mathrm{b}}$ Centre for Health Systems and Safety Research, AIHI, \\ Macquarie University, Australia \\ ${ }^{\mathrm{c}}$ Centre for Medication Safety and Service Quality, Pharmacy Department, \\ Imperial College Healthcare NHS Trust, United Kingdom
}

\begin{abstract}
Evidence is limited of automated dispensing cabinets (ADCs) impact on effectiveness and efficiency of controlled drugs' (CDs) governance processes. We carried out a mixed-methods study of an ADC in an intensive care unit. We found ADC improved effectiveness and efficiency of governance of CDs through secure storage, alerts and automated recording of transactions. Limitations were associated with ADC design and 'fit' between ADC and some types of CDs.
\end{abstract}

Keywords. Controlled drugs, medication systems, hospitals

\section{Introduction}

Opioid-based analgesia and sedatives are potentially dangerous medications, with risks of dependence. The supply and use of these medications are strictly regulated by law. In many countries, legally compliant documentation of stock levels and use of such controlled drugs (CDs) is completed on paper 'registries'. Automated dispensing cabinets (ADCs) can be used in hospital wards to improve efficiency and control of medication supply and use [1]. However, there is a paucity of evidence regarding the impact of ADCs' on CDs. In this poster, we report preliminary findings of an evaluation of a paediatric intensive care unit (PICU) ADC on ward-based processes to control CDs.

\section{Methods}

The study used a mixed-methods design. We carried out $\sim 54$ hours of ethnographic observations in a paediatric intensive care unit (PICU) medication room over 14 days (across June-July 2019) captured as field notes; we observed and timed medication activities in the room using the Work Observation Method By Activity Timing (WOMBAT) tool for a total of $\sim 42$ hours over 13 days in the same period; we conducted

\footnotetext{
${ }^{1}$ Corresponding Author, Valentina Lichtner, UCL School of Pharmacy, BMA House, Tavistock Square, London WC1H 9JP, United Kingdom.
} 
semi-structured interviews with PICU nurses $(n=19)$ and the pharmacy implementation team $(n=4)$; we analyzed ADC transactions logs (March-June 2019); we analyzed all hospital patient safety incidents voluntarily reported in PICU over the three months before and after ADC implementation $(n=171)$ and carried out audits of $C D$ paper registries in PICU and three control wards for the three months before and after implementation (January-June 2019). We carried out a thematic analysis of the qualitative data. The quantitative data were analyzed using descriptive statistics.

\section{Results}

Safekeeping of CDs improved after ADC implementation for CDs kept in ADC double locked drawers. Control of access to CDs also improved through logins and automated recording of all transactions. However, CDs kept in the ADC fridge did not benefit from the same level of control, as the fridge did not support access control for single items.

Documentation of CDs within the ADC decreased opportunities for inaccuracies or errors. Discrepancies in recorded stock level with the ADC were identified in (or near) real time (e.g. through ADC alerts) and all were resolved within 24 hours. Conversely, $\mathrm{CD}$ discrepancies in paper registries remained unnoticed for days or weeks. Un-identified discrepancies tended to accumulate over time and were reported to be more challenging to investigate and address than those identified in (near) real time. Identifying discrepancies in (near) real time had the potential to substantially reduce the burden on pharmacists tasked with the time-consuming process of accounting for discrepancies.

The ADC improved efficiency of governance of CDs. By eliminating the need to retrieve keys to the safe from a nominated nurse, access to CDs (via fingerprint or login) was potentially faster. The task of two nurses performing daily counts of CD stock was reported by nurses to be faster with the ADC than with the safe and registries. The potential of the ADC to improve efficiency of CD governance was limited by some medications (e.g. patients' own CDs and doses of CDs to be destroyed) not being stored in the ADC. This required parallel use of safe and registries as well as the ADC.

\section{Conclusions}

The ADC overall improved effectiveness and efficiency of governance of CDs through secure storage, users' logins, automated recording of transactions, alerts and potential for remote monitoring. There were limitations associated with technology design and with the 'fit' between the ADC and some types of controlled medications.

\section{Acknowledgements}

Funded by the Horizon 2020 Marie Sklodowska-Curie grant agreement No 740131. 


\section{References}

[1] Ahtiainen HK, Kallio MM, Airaksinen M, Holmström A-R. Safety, time and cost evaluation of automated and semi-automated drug distribution systems in hospitals: a systematic review. European Journal of Hospital Pharmacy (2019), ejhpharm-2018-001791. 